February 27, 2018

\title{
New Two-loop Contributions to Hadronic EDMs in the MSSM
}

\author{
Junji Hisano $^{1}$, Minoru Nagai ${ }^{1}$ and Paride Paradisi ${ }^{2,3}$ \\ ${ }^{1}$ ICRR, University of Tokyo, Kashiwa 277-8582, Japan \\ 2 INFN, Sezione di Roma II and Dipartimento di Fisica, \\ Università di Roma "Tor Vergata", I-00133 Rome, Italy \\ ${ }^{3}$ Department of Physics, Technion-Israel Institute of Technology, \\ Technion City, 32000 Haifa, Israel
}

\begin{abstract}
Flavor-changing terms with CP-violating phases in the quark sector may contribute to the hadronic electric dipole moments (EDMs). However, within the Standard Model (SM), the source of $\mathrm{CP}$ violation comes from the unique CKM phase, and it turns out that the EDMs are strongly suppressed. This implies that the EDMs are very sensitive to non-minimal flavor violation structures of theories beyond the SM. In this paper, we discuss the quark EDMs and CEDMs (chromoelectric dipole moments) in the MSSM with general flavorchanging terms in the squark mass matrices. In particular, the charged-Higgs mediated contributions to the down-quark EDM and CEDM are evaluated at two-loop level. We point out that these two-loop contributions may dominate over the one-loop induced gluino or Higgsino contributions even when the squark and gluino masses are around few $\mathrm{TeV}$ and $\tan \beta$ is moderate.
\end{abstract}

\section{Introduction}

Within the Standard Model (SM), the sources of CP violation are the phase in the CKM matrix and the QCD theta $(\bar{\theta})$ parameter. The former induces CP violation in flavorchanging processes, such as $\mathrm{CP}$-violating $K$ and $B$ meson decay modes, whereas the latter induces flavor-conserving $\mathrm{CP}$ violation, such as neutron electric dipole moment (EDM). The experimental upper bound on the neutron EDM gives a strong constraint on $\bar{\theta}$, as $|\bar{\theta}| \lesssim 10^{-(10-11)}$. On the other hand, the recent measurements of CP asymmetries in the $B$ decay modes at the Babar and Belle experiments confirm that the CKM phase is almost maximal and it is the dominant source of $\mathrm{CP}$ violation in the $K$ and $B$ meson decays. 
The minimal supersymmetric SM (MSSM) is the most motivated model beyond the SM. This model exhibits plenty of new CP-violating phases in addition to the unique CKM phase. It is known that they constitute a threat for very sensitive CP tests, at least for SUSY particle masses which are within the TeV region. New CP phases may be introduced in both the flavor-conserving and the flavor-changing soft SUSY breaking terms. The former ones are constrained directly by the electron and hadronic EDMs. In the latter case, flavor-changing neutral current (FCNC) processes are sensitive to those new phases, and we have to satisfy the stringent bounds arising from the $K$ and $B$ physics low-energy experiments.

The strong suppression of the EDMs within the SM and their high sensitivities to physics beyond the SM signal the unique possibilities offered by the EDMs in probing the underlying mechanism of $\mathrm{CP}$ violation. In the present paper, we are interested in the quark EDMs and CEDMs (chromoelectric dipole moments) arising from flavor-changing terms in the squark mass matrices. While the EDMs and CEDMs are highly suppressed within the minimal flavor violation (MFV) flamework, as it happens in the SM, those flavor-changing terms allow us to introduce new types of Jarlskog invariants in the MSSM. The Jarlskog invariants are a basis-independent measure of $\mathrm{CP}$ violation [1]. Within a general flavor violation (GFV) framework, the invariants are less suppressed by the Yukawa coupling constants compared to the MFV case. This implies that the EDMs are sensitive to non-minimal structures of flavor violation. In this paper, we will analyze in detail twoloop effects induced by non-holomorphic Yukawa interactions $[2,3]$ through the effective couplings of down-type quarks/charged-Higgs boson/up-type quarks. Finally, we will also briefly discuss the correlations between quarks CEDMs and $B$ physics observables.

\section{Hadronic EDMs and New Jarlskog Invariants in the MSSM}

The effective CP-violating interactions, which contribute to the hadronic EDMs, up to dimension five operators, are

$$
\mathcal{L}_{C P}=\bar{\theta} \frac{\alpha_{s}}{8 \pi} G \tilde{G}-\sum_{q=u, d, s} i \frac{d_{q}}{2} \bar{q}(F \cdot \sigma) \gamma_{5} q-\sum_{q=u, d, s} i \frac{d_{q}^{c}}{2} \bar{q}\left(g_{s} G \cdot \sigma\right) \gamma_{5} q,
$$

where $F_{\mu \nu}$ and $G_{\mu \nu}$ are the electromagnetic and the $\mathrm{SU}(3)_{C}$ gauge field strengths, respectively [4]. The first term in Eq. (1) is the effective QCD theta term, while $d_{q}$ and $d_{q}^{c}$ are the quark EDM and CEDM, respectively. Within the SM, the sources of CP violation come from the QCD theta term and the CKM matrix, $V$. The theta term is strongly constrained by the experimental bounds on the neutron EDM, as mentioned in the Introduction. One of the most natural ways to achieve such a suppression is to impose a Peccei-Quinn symmetry [5], since the axion field makes $\bar{\theta}$ dynamically vanishing.

Under the above assumption, the hadronic EDMs are strongly suppressed in the SM. The EDMs and CEDMs of the $i$-th down- and up-type quarks are proportional to the 
flavor-conserving Jarlskog invariants,

$$
\begin{aligned}
& J_{\mathrm{SM}}^{\left(d_{i}\right)}=\operatorname{Im}\left\{Y_{d}\left[Y_{d}, Y_{u}\right] Y_{u} f_{d}\right\}_{i i}, \\
& J_{\mathrm{SM}}^{\left(u_{i}\right)}=\operatorname{Im}\left\{Y_{d}\left[Y_{d}, Y_{u}\right] Y_{u} f_{u}\right\}_{i i},
\end{aligned}
$$

respectively. Here, $\left(f_{d}\right)_{i j}\left[\left(f_{u}\right)_{i j}\right]$ is the down[up]-type quark Yukawa coupling constant and $Y_{d(u)} \equiv f_{d(u)} f_{d(u)}^{\dagger}$. The suffixes $i(j)$ in the Yukawa coupling constants refer to left(right)handed quarks. Since the Jarlskog invariants are of the ninth order in the Yukawa coupling constants, the quark (C)EDMs are highly suppressed. The first non-vanishing contributions come from terms of order $O\left(\alpha_{s} G_{F}^{2}\right)$, and $d_{q} / e$ and $d_{q}^{c}$ are $\sim 10^{-(33-34)} \mathrm{cm}$. The neutron EDM may be enhanced by long-distance effects, however, the SM predictions for the hadronic EDMs, including the neutron EDM, are well below the actual and expected future experimental resolution.

The strong suppression of EDMs within a SM framework with the Peccei-Quinn symmetry active, comes from the fact that the CKM contains only one physical phase that is associated, in addition, to the only flavor-changing couplings. However, the above situation may be no longer valid in theories beyond the SM. For instance, within a SUSY framework, various CP-violating couplings may appear after the introduction of SUSY breaking terms. The first ones are the $F$-term SUSY breaking terms, such as the gaugino masses, the $B$ term in the Higgs potential and the $A$ terms for trilinear scalar couplings. The relative phases of the above SUSY breaking terms contribute to the hadronic and electronic EDMs, and the experimental bounds on the EDMs infer strong constraints on their sizes [6]. For instance, barring accidental cancellations among contributions from different phases, it turns out that

$$
\left|\sin \phi_{A}\right| \lesssim\left(\frac{m_{S U S Y}}{\mathrm{TeV}}\right)^{2}, \quad\left|\sin \phi_{B}\right| \lesssim\left(\frac{m_{S U S Y}}{\mathrm{TeV}}\right)^{2} \frac{1}{\tan \beta}
$$

Here, $\phi_{A / B}$ is the relative phase between the $A / B$ parameters and the gaugino masses, and $m_{S U S Y}$ is the typical SUSY particle mass scale.

The problem of so small CP-violating phases is referred to as the SUSY CP problem. Here, we simply assume that the F-term SUSY breaking terms are real. Even under this assumption, we might still have CP-violating phases in the $D$-term SUSY breaking terms, which are sfermion mass terms. The off-diagonal (flavor-changing) terms in the sfermion mass matrices may have CP phases. In the SM, the Jarlskog invariants contributing to the hadronic EDMs are highly suppressed by the structure of the MFV, in which flavor and CP violations come from the only interplays between up- and down-type quark Yukawa couplings. However, in the MSSM we can introduce new invariants even under the assumption that the $F$-term SUSY breaking terms are real. Non-minimal flavor structures in the $D$-terms contribute to new invariants that are, finally, no more suppressed by high powers of the Yukawa coupling constants as it happens, on the contrary, in the SM. This implies that, the hadronic EDMs measurements probe non-minimal flavor structures in the MSSM.

The size and pattern of these flavor violation sources in the sfermion mass matrices depend on the SUSY breaking mechanism and interactions of the high-energy theories 
beyond the MSSM. In the following, the GFV is assumed in the squark mass terms, and we introduce, as usual, the mass insertion parameters defined as

$$
\begin{aligned}
\left(\delta_{L L}^{q}\right)_{i j} & \equiv \frac{\left(m_{\tilde{q}_{L}}^{2}\right)_{i j}}{\bar{m}_{\tilde{q}_{L}}^{2}}, \\
\left(\delta_{R R}^{d}\right)_{i j} & \equiv \frac{\left(m_{\tilde{d}_{R}}^{2}\right)_{i j}}{\bar{m}_{\tilde{d}_{R}}^{2}}, \\
\left(\delta_{R R}^{u}\right)_{i j} & \equiv \frac{\left(m_{\tilde{u}_{R}}^{2}\right)_{i j}}{\bar{m}_{\tilde{u}_{R}}^{2}},
\end{aligned}
$$

for $i \neq j$. The diagonal components of the mass insertion parameters are zero. When calculating the SUSY contribution to the quark EDMs and CEDMs explicitly, we will take a basis in which the down-type quark Yukawa coupling constants are flavor-diagonal. In this case, $\delta_{L L}^{d}=\delta_{L L}^{q}$. In the flavor basis, the mass insertion parameters for left-handed up- and down-type squarks are related by the $\mathrm{SU}(2)_{L}$ symmetry as $\delta_{L L}^{u}=V \delta_{L L}^{d} V^{\dagger}$.

In the MSUGRA model, the renormalization-group ( $R G$ ) effect induced by the topquark Yukawa coupling generates sizable flavor-changing terms in the left-handed downtype squark mass matrix due to the CKM mixing, and it turns out that $\left(\delta_{L L}^{d}\right)_{i j} \propto-V_{3 i}^{*} V_{3 j}$ for moderate $\tan \beta$ values. In the case, the $\delta_{L L}^{u}$ mass insertions are close to zero.

First, let us consider a case of $\left(\delta_{L L}^{q}\right)_{i j} \neq 0$. The new flavor-conserving Jarlskog invariants, which contribute to the quark EDMs and CEDMs, appear at the third order in the Yukawa coupling constants,

$$
\begin{aligned}
& J_{L L}^{\left(d_{i}\right)}=\operatorname{Im}\left\{\left[Y_{u}, \delta_{L L}^{q}\right] f_{d}\right\}_{i i}, \\
& J_{L L}^{\left(u_{i}\right)}=\operatorname{Im}\left\{\left[Y_{d}, \delta_{L L}^{q}\right] f_{u}\right\}_{i i} .
\end{aligned}
$$

$J_{L L}^{\left(d_{i}\right)}$ and $J_{L L}^{\left(u_{i}\right)}$ contribute to the $i$-th down- and up-type quark (C)EDMs. Since chirality of quark is flipped in the quark (C)EDM operators (see Eq. (1D)), the invariants are proportional to the corresponding Yukawa coupling constants. The relative phases between the CKM matrix and $\delta_{L L}^{q}$ contribute to the invariants. In the MSUGRA model, non-zero values for $\left(\delta_{L L}^{q}\right)_{i j}$ are induced by the RG effect, and they turn out to be proportional to $\left(Y_{u}\right)_{i j}$ or $\left(Y_{d}\right)_{i j}$. However, those flavor structures are not able to contribute to the Jarlskog invariants. This implies that only non-minimal flavor structures generate the quark (C)EDMs via the new invariants, as stressed above.

Higgsino diagrams may contribute to the (C)EDMs at one-loop level in the case of $\left(\delta_{L L}^{q}\right)_{i j} \neq 0$ (see Fig. 1), since $\delta_{L L}^{q}$ is sandwiched between the Yukawa coupling constants in the invariants [7]. The down-quark CEDM and EDM are larger than those of up quark for moderate $\tan \beta$, since $J_{L L}^{(u)}$ is suppressed by the square of down-type quark Yukawa coupling constants. In the limit of degenerate SUSY particle masses, it is found that the down- and strange-quark EDMs and CEDMs are given as

$$
\left\{\frac{\left(d_{d_{i}}\right)_{\tilde{H}^{ \pm}}}{e},\left(d_{d_{i}}^{c}\right)_{\tilde{H}^{ \pm}}\right\}=-\frac{\alpha_{2}}{4 \pi}\left(\frac{m_{d_{i}}}{m_{W}^{2}}\right)\left(\frac{m_{t}^{2}}{m_{\tilde{q}}^{2}}\right)\left(\frac{\mu A_{t}}{m_{\tilde{q}}^{2}}\right) \tan \beta\left[\operatorname{Im}\left\{\left(\delta_{L L}^{u}\right)_{i 3} V_{3 i}\right\}\right]\left\{\frac{1}{30}, \frac{1}{40}\right\},
$$



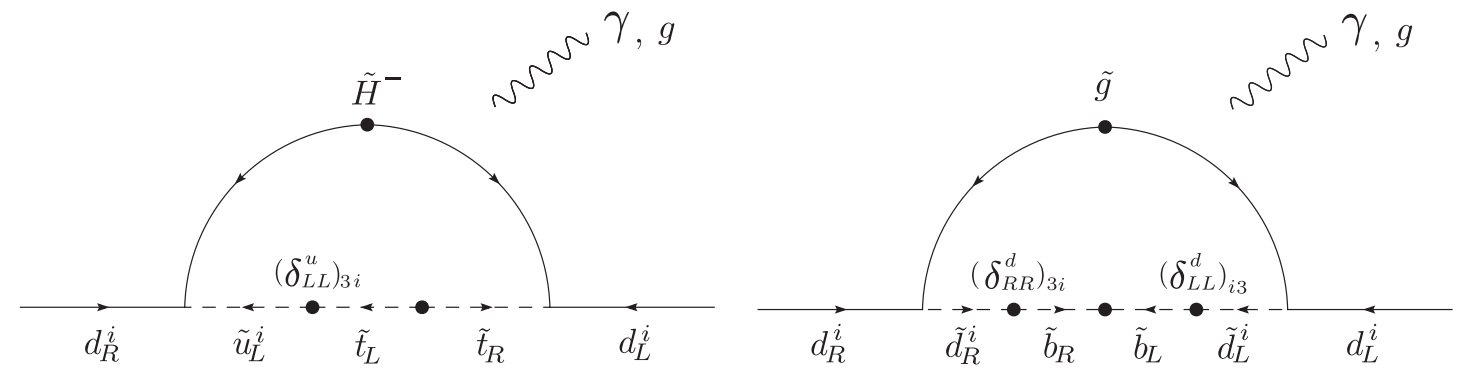

Figure 1: Left: Higgsino contribution to the $i$-th down-quark (C)EDM in a case of nonvanishing left-handed squark mixing, $\left(\delta_{L L}^{q}\right)_{3 j} \neq 0$. Right: Gluino contribution to the $i$-th down-quark $(\mathrm{C}) \mathrm{EDM}$ in a case of non-zero values for both left- and right-handed squark mixings, $\left(\delta_{L L}^{q}\right)_{i 3} \neq 0$ and $\left(\delta_{R R}^{d}\right)_{3 i} \neq 0$.

where $i=1,2$. The $(\mathrm{C})$ EDMs are suppressed by the external light quark masses, as expected from the invariants (5). For $\left|\left(\delta_{L L}^{q}\right)_{13}\right| \sim \lambda^{3}, m_{S U S Y}=500 \mathrm{GeV}$ and $\tan \beta=10$, $d_{d} / e$ and $d_{d}^{c}$ are of order $\sim 10^{-28} \mathrm{~cm}$.

When $\left(\delta_{R R}^{d}\right)_{i j} \neq 0$ or $\left(\delta_{R R}^{u}\right)_{i j} \neq 0$, the new Jarlskog invariants are

$$
\begin{aligned}
& J_{R R}^{\left(d_{i}\right)}=\operatorname{Im}\left\{Y_{u} f_{d} \delta_{R R}^{d}\right\}_{i i}, \\
& J_{R R}^{\left(u_{i}\right)}=\operatorname{Im}\left\{Y_{d} f_{u} \delta_{R R}^{u}\right\}_{i i} .
\end{aligned}
$$

In the above cases, no diagram contributes to the quark (C)EDMs at one-loop level, since the mass insertions appear in the right-hand side of the Yukawa coupling constants in the invariants. The first non-vanishing contribution arises from higher-order effects (two-loop effects), and the quark (C)EDMs are suppressed by loop factors. However, the (C)EDMs may be enhanced by the Yukawa coupling constant of the heaviest generation via the CKM and right-handed squark mixings, that partially compensates the loop suppression. In the next section, we will show that the non-holomorphic Yukawa coupling for $\bar{u}_{L i} d_{R j} H^{+}$vertex is generated at one-loop level and is proportional to $f_{d_{i}}\left(\delta_{R R}^{d}\right)_{i j}$ in the cases of $\left(\delta_{R R}^{d}\right)_{i j} \neq 0$. Thus, the charged-Higgs loop contributes to the down-quark (C)EDM at two-loop level, providing a contribution proportional to $J_{R R}^{(d)}$.

In a case of both left- and right-handed squarks have mixings, the (C)EDMs are derived at one-loop level and are also enhanced by the heaviest quark masses. The new Jarlskog invariants are

$$
\begin{gathered}
J_{L R}^{\left(d_{i}\right)}=\operatorname{Im}\left\{\delta_{L L}^{q} f_{d} \delta_{R R}^{d}\right\}_{i i} \\
J_{L R}^{\left(u_{i}\right)}=\operatorname{Im}\left\{\delta_{L L}^{q} f_{u} \delta_{R R}^{u}\right\}_{i i} .
\end{gathered}
$$

The above invariants are proportional to only one Yukawa coupling constant, and the relative phases of the left- and right-handed squark mixings contribute to them.

The gluino diagrams in Fig. 1 contribute to the quark (C)EDMs, being proportional 
to the above $J_{L R}^{\left(d_{i}, u_{i}\right)}$ invariants ${ }^{1}$. The down- and strange-quark EDMs and CEDMs are given as

$$
\left\{\frac{\left(d_{d_{i}}\right)_{\tilde{g}}}{e},\left(d_{d_{i}}^{c}\right)_{\tilde{g}}\right\}=-\frac{\alpha_{s}}{4 \pi} \frac{m_{b} \mu \tan \beta}{m_{\tilde{q}}^{3}}\left[\operatorname{Im}\left\{\left(\delta_{L L}^{d}\right)_{i 3}\left(\delta_{R R}^{d}\right)_{3 i}\right\}\right]\left\{\frac{4}{135}, \frac{11}{180}\right\},
$$

where $i=1,2$. In the above expression we have set the gluino mass $m_{\tilde{g}}$ to be equal to the squark masses $m_{\tilde{q}}$. The down-quark EDM reaches to the $10^{-(25-26)} \mathrm{e} \mathrm{cm}$ level even for $m_{S U S Y}=500 \mathrm{GeV},\left(\delta_{L L}^{d}\right)_{13} \sim\left(\delta_{R R}^{d}\right)_{31} \sim \lambda^{3}$ and $\tan \beta=10$. Thus, in this case, the current experimental bounds on the hadronic EDMs give constraints on the model parameters. SUSY GUTs predict both left-handed and right-handed squark mixings, and the related one-loop induced EDMs have been studied in many papers [8].

In the next section, we will introduce the non-holomorphic Yukawa couplings of the charged-Higgs boson and evaluate the down-quark (C)EDM at two-loop level when $\left(\delta_{L L}^{q}\right)_{i j} \neq 0$ or $\left(\delta_{R R}^{d}\right)_{i j} \neq 0$. Other cases where $\left(\delta_{R R}^{u}\right)_{i j} \neq 0$ are remanded to our future works. The up-quark (C)EDM from the charged-Higgs loop is proportional to tan $\beta$ while $J_{R R}^{(u)}$ is proportional to $\tan ^{2} \beta$. This implies that we need a more complete analysis since other two-loop diagrams may dominate over it.

\section{Non-holomorphic Yukawa Interactions and Quark (C)EDMs}

In the present paper, we are mainly interested in possible $\mathrm{CP}$-violating effects arising from the Higgs sector through non-holomorphic Yukawa interactions that we are going to discuss. When $\left(\delta_{R R}^{d}\right)_{3 i} \neq 0(i=1,2)$, the effective $\bar{t}_{L} d_{R}^{i} H^{+}$coupling is derived from the wave function renormalization of right-handed down-type quarks as shown in the Feynman diagram of Fig. 2. and it is enhanced by the bottom-quark mass. The charged-Higgs loop contributes to the down- and strange-quark (C)EDMs that result to be proportional to the Jarlskog invariants $J_{R R}^{(d, s)}$, as expected. Given that the above contributions are generated by non-holomorphic Yukawa interactions, they do not decouple even when the SUSY particle masses are very heavy ${ }^{2}$.

Following the procedure of Refs. $[11,12]$, in order to compute all the non-decoupling effects at large $\tan \beta$ one needs to: $i$ ) evaluate the effective dimension-four operators appearing at one-loop level which modify the tree-level Yukawa Lagrangian; ii) expand the off-diagonal mass terms in the squark sector by means of the mass-insertion approximation; iii) diagonalize the quark mass terms and derive the effective interactions between quarks and heavy Higgs fields.

\footnotetext{
${ }^{1}$ The bino diagrams similar to Fig. [1 also contribute to the quark (C)EDMs, being proportional to the above $J_{L R}^{\left(d_{i}, u_{i}\right)}$ invariants. However, they are always sub-dominant compared to the gluino ones.

${ }^{2}$ As recently shown in Ref. [9,10], charged-Higgs boson can induce other very interesting non-standard phenomena in the $K$ meson system: violations of lepton universality which can reach the $1 \%$ level in the $\Gamma(K \rightarrow e \nu) / \Gamma(K \rightarrow \mu \nu)$ ratio [9] and large deviation from the SM expectation in the branching ratios of $K \rightarrow \pi \nu \bar{\nu}[10]$.
} 
The effective interaction of $\bar{t}_{L} d_{R}^{i} H^{+}$and $\bar{t}_{R} d_{L}^{i} H^{+}$is parametrized as

$$
\begin{aligned}
\mathcal{L}_{H^{ \pm}}^{\mathrm{eff}} & =\frac{g}{\sqrt{2} m_{W}} \frac{m_{t}}{\tan \beta} V_{3 i} C_{L}^{H^{+}} \bar{t}_{R} d_{L}^{i} H^{+} \\
& +\frac{g}{\sqrt{2} m_{W}} m_{d_{i}} \tan \beta V_{3 i} C_{R}^{H^{+}} \bar{t}_{L} d_{R}^{i} H^{+}+\text {h.c.. }
\end{aligned}
$$

The dominant corrections to the charged-Higgs vertex arise from gluino exchange, and the effective charged-Higgs coupling constants are given by

$$
\begin{gathered}
C_{L}^{H^{+}}=\left(1-\epsilon_{s} \tan \beta\right)+\epsilon_{f} \frac{\left(\delta_{L L}^{d}\right)_{3 i}}{V_{3 i}} \tan \beta \\
C_{R}^{H^{+}}=\frac{1}{\left(1+\epsilon_{s} \tan \beta\right)}\left(1-\frac{\epsilon_{f} \tan \beta}{\left(1+\epsilon_{s} \tan \beta\right)} \frac{\left(\delta_{L L}^{d}\right)_{3 i}}{V_{3 i}}+\frac{\epsilon_{f} \tan \beta}{\left(1+\epsilon_{s} \tan \beta\right)} \frac{m_{b}}{m_{d_{i}}} \frac{\left(\delta_{R R}^{d}\right)_{3 i}}{V_{3 i}}\right) .
\end{gathered}
$$

The sub-dominant Higgsino contributions are neglected. They become relevant only for very large values of $\tan \beta$, i.e. $\tan \beta \gtrsim 40$. Here, $V$ refers to the renormalized CKM matrix at one-loop level. When $\tan \beta$ is quite large, the radiative corrections to the CKM matrix are not negligible. The elements of the renormalized CKM matrix, which are relevant in the following discussion, are given from the tree-level ones $\left(V^{\text {tree }}\right)$ as

$$
V_{3 i}=V_{3 i}^{\text {tree }}+\frac{\epsilon_{f} \tan \beta}{1+\epsilon_{s} \tan \beta}\left(\delta_{L L}^{d}\right)_{3 i}
$$

In the above equations, the $\epsilon_{s}$ and $\epsilon_{f}$ terms are given by

$$
\epsilon_{s}=\frac{\alpha_{s}}{3 \pi} \frac{\mu}{m_{\tilde{q}}}, \quad \epsilon_{f}=\frac{\alpha_{s}}{9 \pi} \frac{\mu}{m_{\tilde{q}}} .
$$

Here, we assumed that the squark masses $m_{\tilde{q}}$ are equal to the gluino mass $m_{\tilde{g}}$.

It is noticed that the effective coupling in Eq. (12) contains terms not suppressed by the $m_{d_{i}}(i=1,2)$ factor. Thanks to the right-handed squark mixing terms, we can always pick up the bottom mass $m_{b}$ instead of $m_{d}$ or $m_{s}$. We stress that, in the MFV framework, it is not possible to get such a Yukawa enhancement.

The (C)EDMs of down and strange quarks are induced by the one-loop exchange of the charged-Higgs boson and top quark, as it is shown in Fig. 2. By making use of the effective Lagrangian in Eq. (10), they are evaluated as

$$
\begin{aligned}
\left\{\frac{\left(d_{d_{i}}\right)_{H^{ \pm}}}{e},\left(d_{d_{i}}^{c}\right)_{H^{ \pm}}\right\}= & \frac{\alpha_{2}}{4 \pi}\left|V_{3 i}\right|^{2}\left(\frac{m_{d_{i}}}{m_{W}^{2}}\right)\left(\frac{m_{t}^{2}}{m_{H^{ \pm}}^{2}}\right)\left[\operatorname{Im}\left\{C_{L}^{H^{+}} C_{R}^{H^{+}}\right\}\right] \\
& \times\left\{F_{7}, F_{8}\right\}\left(y_{t H}\right),
\end{aligned}
$$

where $y_{t H}=m_{t}^{2} / m_{H^{ \pm}}^{2}$ and the loop functions $F_{7}(x)$ and $F_{8}(x)$ are given by

$$
F_{7}(x)=\frac{3-5 x}{12(1-x)^{2}}-\frac{3 x-2}{6(1-x)^{3}} \log (x), \quad F_{8}(x)=\frac{3-x}{4(1-x)^{2}}+\frac{\log (x)}{2(1-x)^{3}} .
$$



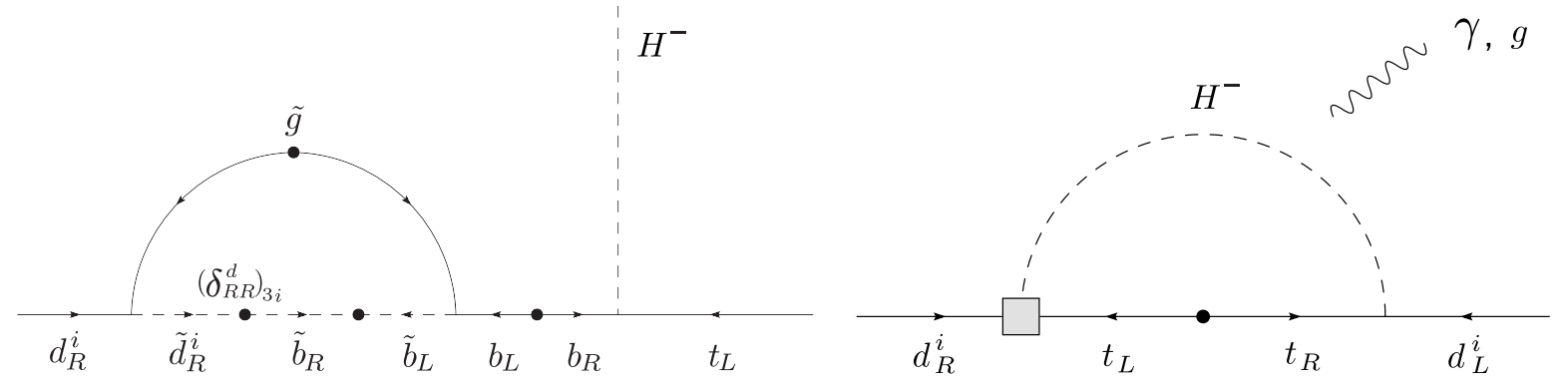

Figure 2: Left: Leading contribution to the effective $\bar{t}_{L} d_{R}^{i} H^{+}$coupling in Eq. (12) in a case of $\left(\delta_{R R}^{d}\right)_{i 3} \neq 0$. Right: Charged-Higgs mediated diagrams contributing to the (C)EDMs in a case of $\left(\delta_{R R}^{d}\right)_{i 3} \neq 0$.

In the case of $\left(\delta_{R R}^{d}\right)_{i 3} \neq 0(i=1,2)$, the charged-Higgs contributions to the (C)EDMs are given as

$$
\begin{aligned}
\left\{\frac{\left(d_{d_{i}}\right)_{H^{ \pm}}}{e},\left(d_{d_{i}}^{c}\right)_{H^{ \pm}}\right\}= & -\frac{\alpha_{2}}{4 \pi}\left[\operatorname{Im}\left\{V_{3 i}^{\star}\left(\delta_{R R}^{d}\right)_{3 i}\right\}\right]\left(\frac{m_{b}}{m_{W}^{2}}\right)\left(\frac{m_{t}^{2}}{m_{H^{ \pm}}^{2}}\right) \frac{\epsilon_{f}\left(1-\epsilon_{s} \tan \beta\right) \tan \beta}{\left(1+\epsilon_{s} \tan \beta\right)^{2}} \\
& \times\left\{F_{7}, F_{8}\right\}\left(y_{t H}\right),
\end{aligned}
$$

while in the case of $\left(\delta_{L L}^{d}\right)_{i 3} \neq 0(i=1,2)$

$$
\begin{aligned}
\left\{\frac{\left(d_{d_{i}}\right)_{H^{ \pm}}}{e},\left(d_{d_{i}}^{c}\right)_{H^{ \pm}}\right\}= & -\frac{\alpha_{2}}{4 \pi}\left[\operatorname{Im}\left\{\left(\delta_{L L}^{d}\right)_{i 3} V_{3 i}\right\}\right]\left(\frac{m_{d_{i}}}{m_{W}^{2}}\right)\left(\frac{m_{t}^{2}}{m_{H^{ \pm}}^{2}}\right) \frac{2 \epsilon_{f} \tan \beta}{\left(1+\epsilon_{s} \tan \beta\right)^{2}} \\
& \times\left\{F_{7}, F_{8}\right\}\left(y_{t H}\right) .
\end{aligned}
$$

The above contributions are proportional to $J_{R R}^{\left(d_{i}\right)}$ and $J_{L L}^{\left(d_{i}\right)}$, respectively. Making a comparison with the contributions in Eq. (17), it is observed that the effects in Eq. (18) are suppressed by a $m_{d_{i}} / m_{b}$ factor, as expected.

In principle, the exchange of the neutral-Higgs bosons, $H^{0}$ and $A^{0}$, could also contribute to the (C)EDMs. Their contributions are enhanced by a $\tan ^{4} \beta$ factor, due to the nature of the non-holomorphic Yukawa couplings [3], while they suffer from a suppression of order $\left(m_{b} / m_{t}\right)^{2}$, compared to the charged-Higgs case. However, these effects arise at the three-loop level and are additionally reduced by a cancellation between the $A^{0}$ and $H^{0}$ contributions (due to the mass degeneration of $A^{0}$ and $H^{0}$ at tree level). We found that the neutral-Higgs effects to the (C)EDMs are largely sub-dominant compared to the charged-Higgs ones even in the most favorable circumstance, namely for large $\tan \beta$ values and for moderate mass splitting between $H^{0}$ and $A^{0}\left(\delta m / m_{A^{0}} \sim 10 \%\right)$.

\section{Numerical analysis}

The sensitivity of the down-quark (C)EDM to the charged-Higgs mediated effects is illustrated on the left of Fig. [3. In this figure, we assume $\left|\left(\delta_{R R}^{d}\right)_{31}\right|=\lambda^{3}$. From this figure, 

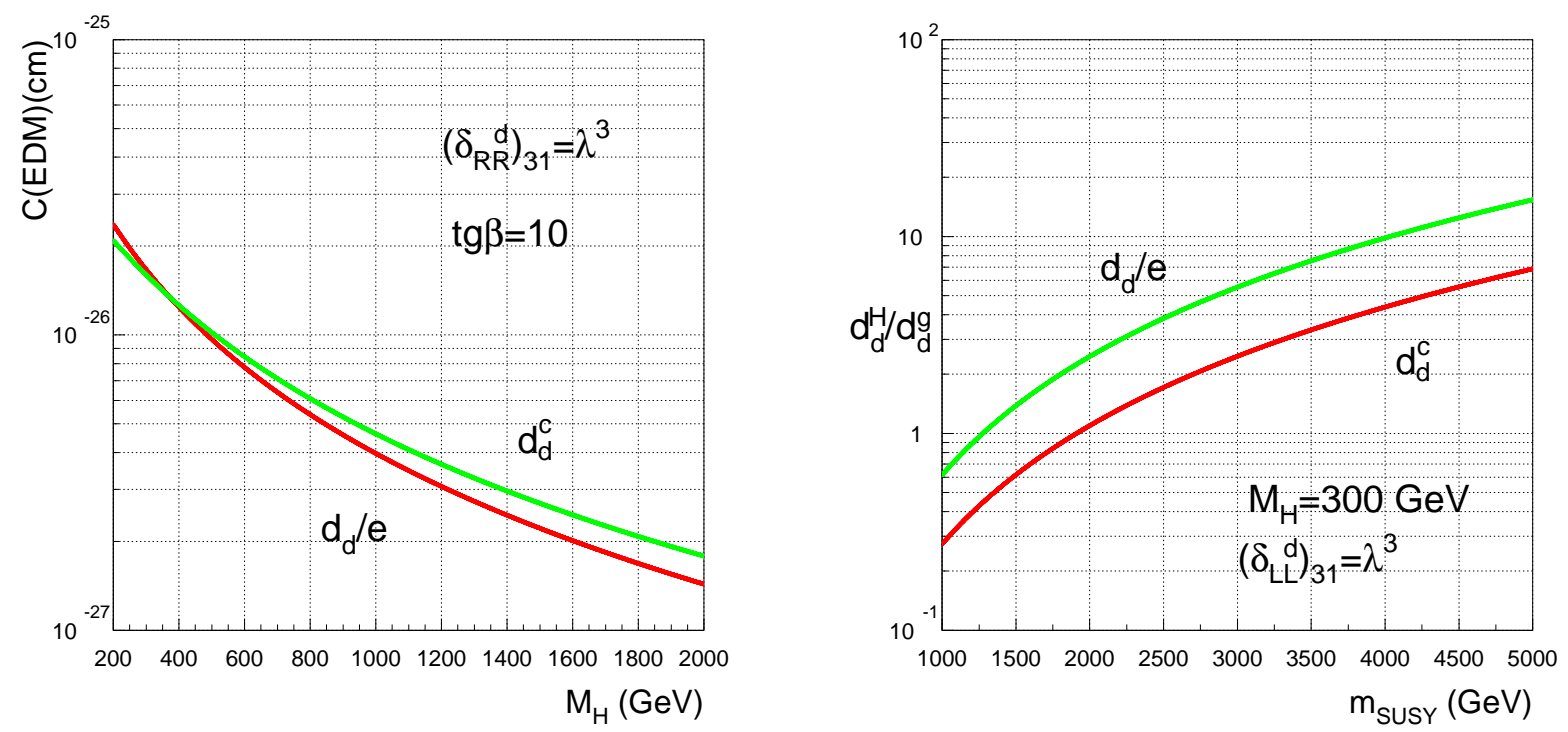

Figure 3: Left: Down-quark EDM and CEDM as functions of the charged-Higgs mass $m_{H^{ \pm}}$for $\tan \beta=10$. The (C)EDM scales as $\tan \beta / 10$. In this figure, a CKM-type mixing is assumed for $\delta_{R R}^{d}$, i.e. $\left|\left(\delta_{R R}^{d}\right)_{31}\right|=\lambda^{3}$. Right: Ratio of charged-Higgs and gluino induced down-quark (C)EDM as a function of a common SUSY particle mass $m_{S U S Y}$, setting the Higgs mass to $m_{H^{ \pm}}=300 \mathrm{GeV}$. We assume $\left(\delta_{L L}^{d}\right)_{13}=-V_{31}^{\star}$.

$\left(d_{d}^{c}\right)_{H^{ \pm}}$and $\left(d_{d} / e\right)_{H^{ \pm}}$can be of order $10^{-(26-27)} \mathrm{cm}$ for $\tan \beta=10$ and $m_{H^{ \pm}} \in(0.2,2) \mathrm{TeV}^{3}$. On the other hand, the current experimental upper bounds on the EDMs of neutron, $\left|d_{n}\right|<3.0 \times 10^{-26} e \mathrm{~cm}$ [13], and of ${ }^{199} \mathrm{Hg}$ atom, $\left|d_{\mathrm{Hg}}\right|<2.1 \times 10^{-28} e \mathrm{~cm}$ [14], constrain the down-quark EDM and CEDM as $\left|d_{d}^{c}\right|$ and $\left|d_{d}\right| / e \lesssim 10^{-26} \mathrm{~cm}$, barring accidental cancellations among contributions [4] [15]. The charged-Higgs mediated effects to the (C)EDM are very close to the actual experimental sensitivities and will be strongly probed with the expected future experimental resolutions.

The strange-quark CEDM is easily obtained by means of the down-quark CEDM in the following way,

$$
\frac{d_{s}^{c}}{d_{d}^{c}}=\frac{\operatorname{Im}\left\{V_{32}^{\star}\left(\delta_{R R}^{d}\right)_{32}\right\}}{\operatorname{Im}\left\{V_{31}^{\star}\left(\delta_{R R}^{d}\right)_{31}\right\}} .
$$

The neutron EDM might receive a sizable contribution from the strange-quark CEDM as $d_{n} \sim 0.1 e d_{s}^{c}[15,16]$, while the QCD uncertainties are still not well enough under control [4]. When the neutron EDM bound gives a significant constraint on the strange-quark CEDM, it is transmitted as a bound on $\left(\delta_{R R}^{d}\right)_{32}$.

When the SUSY scenario contains only the left-handed squark mixings, the expected values for the down-quark EDM and CEDM are reduced by a $m_{d} / m_{b}$ factor compared to

\footnotetext{
${ }^{3}$ In the following, we take $\operatorname{sign}(\mu A)<0$, as it is preferred by the $(g-2)_{\mu}$ and $b \rightarrow s \gamma$ experimental results.
} 
the case of Fig. 3. They can reach to the level of $10^{-28}(e) \mathrm{cm}$, which is comparable to the one-loop Higgsino contribution, given in Eq. (6).

A non-trivial constraint on the charged-Higgs mass is extracted from $\Gamma\left(B \rightarrow X_{s} \gamma\right)$. It is well known that, the charged-Higgs and chargino contributions to this observable must have opposite sign in order to fulfill its precise experimental determination, when the chargino and charged Higgs masses are not heavy enough to be decoupled. The relative sign between these two amplitudes is proportional to $\operatorname{sign}\left(\mu A_{t}\right)$, where $A_{t}$ is the trilinear soft-breaking term appearing in the stop mass matrix. However, if the SUSY particles are heavy (above the $\mathrm{TeV}$ scale), the chargino contributions are not so effective, and $\Gamma\left(B \rightarrow X_{s} \gamma\right)$ gets the dominant effect by the charged-Higgs contributions. In such cases, it turns out that $m_{H^{ \pm}} \gtrsim 300 \mathrm{GeV}$ [17]. This lower bound on the charged-Higgs mass may be more relaxed (below $m_{H^{ \pm}} \sim 300 \mathrm{GeV}$ ) in the large $\tan \beta$ regime, since sizable higherorder (non-holomorphic) effects interfere destructively with the leading contributions [18].

An interesting feature of the charged-Higgs contributions to the (C)EDMs is the slow decoupling for $m_{H^{ \pm}} \rightarrow \infty$, in contrast to the one-loop gluino or Higgsino contributions (Fig. 1). Being generated by effective one-loop diagrams, the $F_{7,8}\left(y_{t H}\right)$ loop functions develop a large logarithm so that the (C)EDMs behave as $\left(d_{q}^{(c)}\right)_{H^{ \pm}} \sim y_{t H} \log \left(y_{t H}\right)$ for $y_{t H}=$ $m_{t}^{2} / m_{H^{ \pm}}^{2} \ll 1$. This property is clearly shown in Fig. 3. where to one order of magnitude increase of the Higgs mass corresponds only a 10 factor reduction of the (C)EDMs. As a result, even for moderate $\tan \beta$ values and charged-Higgs masses $m_{H^{ \pm}}<2 \mathrm{TeV}$, the down-quark (C)EDM remains at a potential visible level, i.e. $\left(d_{d} / e\right)_{H^{ \pm}}$and $\left(d_{d}^{c}\right)_{H^{ \pm}} \sim$ $\mathcal{O}\left(10^{-(26-27)}\right) \mathrm{cm}$ for $\left|\left(\delta_{R R}^{d}\right)_{31}\right|=\lambda^{3}$.

Next, let us compare the two-loop charged-Higgs contributions in Eq. (17) to the one-loop gluino ones (Fig. 1), in the case of $\left(\delta_{R R}^{d}\right)_{3 i} \neq 0$. Assuming the simple case of degenerate SUSY particle masses, one gets

$$
\begin{aligned}
\left\{\frac{\left(d_{d_{i}}\right)_{H^{ \pm}}}{\left(d_{d_{i}}\right)_{\tilde{g}}}, \frac{\left(d_{d_{i}}^{c}\right)_{H^{ \pm}}}{\left(d_{d_{i}}^{c}\right)_{\tilde{g}}}\right\} \simeq & \frac{\alpha_{2}}{9 \pi}\left(\frac{m_{t}^{2}}{m_{W}^{2}}\right)\left(\frac{m_{\tilde{q}}^{2}}{m_{H^{ \pm}}^{2}}\right) \frac{\operatorname{Im}\left\{\left(\delta_{L L}^{d}\right)_{i 3}\left(\delta_{R R}^{d}\right)_{3 i}\right\}}{\operatorname{Im}\left\{V_{3 i}^{\star}\left(\delta_{R R}^{d}\right)_{3 i}\right\}} \\
& \times\left\{\frac{135}{4} F_{7}, \frac{180}{11} F_{8}\right\}\left(y_{t H}\right) .
\end{aligned}
$$

with $F_{7} \in(-0.24,-1.41)$ and $F_{8} \in(-0.21,-1.76)$ when $m_{H^{ \pm}} \in(0.2,2) \mathrm{TeV}$. Here, we assumed moderate $\tan \beta$ values and ignored higher-order terms of $\alpha_{s}$. The plot on the right of Fig. 3 shows the ratio between charged-Higgs and gluino contributions to the downquark (C)EDM as a function of the squark mass. In the figure, we take $\left(\delta_{L L}^{d}\right)_{13}=-V_{31}^{\star}$. The charged-Higgs mediated effects do not vanish even in the limit of heavy squarks and gluinos, provided the Higgs sector remains relatively light. As a result, the two-loop Higgs mediated (C)EDMs are larger than the one-loop gluino mediated ones if $m_{S U S Y} \gtrsim$ $(1-2) \mathrm{TeV}$ when $m_{H^{ \pm}} \sim 300 \mathrm{GeV}$.

Noteworthy, it is found from Eq. (20) that the ratios of charged-Higgs and gluino contributions are independent of the $\mu$ sign and also on $\tan \beta$, for moderate $\tan \beta$ values. When the non-zero values of $\left(\delta_{L L}^{d}\right)_{i 3}$ come from the RG effect due to the top-quark Yukawa 
coupling as in the MSUGRA model, it is proportional to $-V_{3 i}^{*}$. Thus, the one- and twoloop effects are constructive.

When the SUSY framework contains the only flavor structures in the left-handed squark mass terms, the ratio of the charged-Higgs and Higgsino contributions to the down- and strange-quark (C)EDMs are

$$
\begin{aligned}
\left\{\frac{\left(d_{d_{i}}\right)_{H^{ \pm}}}{\left(d_{d_{i}}\right)_{\tilde{H}^{ \pm}}}, \frac{\left(d_{d_{i}}^{c}\right)_{H^{ \pm}}}{\left(d_{d_{i}}^{c}\right)_{\tilde{H}^{ \pm}}}\right\} \simeq & \frac{2 \alpha_{s}}{9 \pi}\left(\frac{m_{\tilde{q}}^{2}}{m_{H^{ \pm}}^{2}}\right)\left(\frac{A_{t}}{m_{\tilde{q}}}\right)^{-1} \frac{\operatorname{Im}\left\{\left(\delta_{L L}^{d}\right)_{i 3} V_{3 i}\right\}}{\operatorname{Im}\left\{\left(\delta_{L L}^{u}\right)_{i 3} V_{3 i}\right\}} \\
& \times\left\{30 F_{7}, 40 F_{8}\right\}\left(y_{t H}\right) .
\end{aligned}
$$

Reminding that $\delta_{L L}^{u}=V \delta_{L L}^{d} V^{\dagger}$ because of the $\mathrm{SU}(2)_{L}$ symmetry, it turns out that $\left(\delta_{L L}^{u}\right)_{13} \sim\left(\delta_{L L}^{d}\right)_{13}+\lambda\left(\delta_{L L}^{d}\right)_{23}$ and $\left(\delta_{L L}^{u}\right)_{23} \sim\left(\delta_{L L}^{d}\right)_{23}-\lambda\left(\delta_{L L}^{d}\right)_{13}$. From Eq. (21) it is straightforward to check that, barring accidental cancellations among contributions arising from different flavor structures, the charged-Higgs effects to down- and strange-quark (C)EDMs dominate over the chargino ones even if $m_{S U S Y}$ is few times larger than $m_{H^{ \pm}}$. Thus, the charged-Higgs contribution should be included in the case that only the lefthanded squark matrix has non-minimal flavor structures.

\section{Conclusions and Discussion}

In this paper, we have discussed the quark EDMs and CEDMs in the MSSM with GFV terms in the squark mass matrices. In the SM, where the only source of CP violation comes from the CKM matrix, the EDMs are strongly suppressed by high powers of the Yukawa coupling constants. This implies that the EDMs may be very sensitive to nonminimal flavor structures of theories beyond the SM, such as the MSSM. In the MSSM, the first contributions to the (C)EDMs are generated at one-loop level by exchange of Higgsino/squarks or gluino/squarks. While in the former case, non-vanishing left-handed squark mixings are enough to generate the EDMs, in the latter case both left and righthanded squarks mixings are required.

In this work, we have pointed out that new two-loop contributions mediated by the charged-Higgs exchange may generate the quark (C)EDMs. In particular, we have evaluated the down- and strange-quark EDMs and CEDMs. Interesting enough, the above contributions do not decouple even for heavy squarks and gluino. They are proportional to $\tan \beta$ for moderate $\tan \beta$, similar to the one-loop contributions. These new contributions may dominate over the one-loop contributions in the case of non-vanishing right-handed down-type squark mixing, even when the squark and gluino masses are around the TeV scale. Furthermore, the down-quark EDM and CEDM may be very close to the actual experimental sensitivities, since $d_{d} / e$ and $d_{d}^{c} \sim \mathcal{O}\left(10^{-(26-27)}\right) \mathrm{cm}$ for $\left|\left(\delta_{R R}^{d}\right)_{31}\right|=\lambda^{3}$. They will be strongly probed with the expected future experimental resolutions.

In the case that the only left-handed squark mixings are non-vanishing, the two-loop contributions may dominate over the one-loop Higgsino ones even when SUSY particle masses are lighter than $1 \mathrm{TeV}$. Thus, the two-loop contribution cannot be neglected. The 
down-quark EDM and CEDM may reach the level of $\sim \mathcal{O}\left(10^{-28}\right)(e) \mathrm{cm}$ for $\left|\left(\delta_{L L}^{q}\right)_{13}\right| \sim \lambda^{3}$, and they might be also covered in the future experiments.

At large $\tan \beta$, the observable EDMs of neutron and heavy atoms receive contributions not only from the (C)EDMs of the constituent particles, such as electrons and quarks, but also from CP-odd four-fermion operators through the exchange of neutral-Higgs bosons [19]. These effects would be sensitive to CP phases in the flavor-diagonal F-term SUSY breaking terms. We also noticed that within the GFV framework, the phases in the squark mass matrices might also provide a contribution to the $\mathrm{CP}$-odd four-fermion operators. This happens when both left-handed and right-handed squarks have mixing. However, we found that these contributions are smaller than those arising from the charged-Higgs exchange, at least for moderate $\tan \beta$.

Finally, we discuss a correlation between the hadronic EDMs and FCNC processes. In Ref. [20], it has been pointed out that, within some SUSY models, $d_{s}^{c}$ and the deviation from the SM prediction of the CP asymmetry in $B \rightarrow \phi K_{S}\left(\delta S_{\phi K_{s}}\right)$ are correlated. Nonvanishing right-handed down-type squark mixing $\left(\delta_{R R}^{d}\right)_{23}$ may induce sizable $\delta S_{\phi K_{s}}$ via the gluino penguin diagram. The effective Lagrangian relevant for the penguin diagrams is $\mathcal{L}^{\text {eff }}=g_{s} m_{b} /\left(16 \pi^{2}\right) C_{8}^{L} \bar{s}_{L}(G \cdot \sigma) b_{R}+g_{s} m_{b} /\left(16 \pi^{2}\right) C_{8}^{R} \bar{s}_{R}(G \cdot \sigma) b_{L}$, and the right-handed squark mixing contributes to $C_{8}^{R}$. If we additionally require that $\left(\delta_{L L}^{d}\right)_{23} \neq 0$, such as in the MSUGRA model, it turns out that $d_{s}^{c}$ and $\delta S_{\phi K_{s}}$ are correlated, since

$$
\left(d_{s}^{c}\right)_{\tilde{g}}=\frac{m_{b}}{8 \pi^{2}} \frac{11}{21} \operatorname{Im}\left\{\left(\delta_{L L}^{d}\right)_{32}\left(C_{8}^{R}\right)_{\tilde{g}}\right\}
$$

up to the QCD correction.

A similar correlation also appears when the the charged-Higgs mediation effect is the dominant source for $\delta S_{\phi K_{s}}$. In the case of $\left(\delta_{R R}^{d}\right)_{23} \neq 0$,

$$
\left(d_{s}^{c}\right)_{H^{ \pm}}=\frac{m_{b}}{8 \pi^{2}} \operatorname{Im}\left\{V_{32}\left(C_{8}^{R}\right)_{H^{ \pm}}\right\} .
$$

Then, in the charged-Higgs mediated case, $\delta S_{\phi K_{s}}$ is also constrained by the hadronic EDMs, when the hadronic EDMs receive sizable contributions from the strange-quark CEDM. A similar discussion applies also to $b \rightarrow s / d+\gamma$ and so on. A detailed analysis of these last effects is remanded to our future works.

\section{Acknowledgments}

The work of J.H. is supported in part by the Grant-in-Aid for Science Research, Ministry of Education, Science and Culture, Japan (No. 1554055 and No. 18034002). Also, that of M.N. is supported in part by JSPS.

\section{References}

[1] C. Jarlskog, Phys. Rev. Lett. 55 (1985) 1039. 
[2] L.J. Hall, R. Rattazzi and U. Sarid, Phys. Rev. D 50 (1994) 7048.

[3] C. Hamzaoui, M. Pospelov and M. Toharia, Phys. Rev. D 59 (1999) 59; K. S. Babu and C. F. Kolda, Phys. Rev. Lett. 84 (2000) 228.

[4] For a review of EDMs, M. Pospelov and A. Ritz, Annals Phys. 318 (2005) 119.

[5] R. D. Peccei and H. R. Quinn, Phys. Rev. Lett. 38 (1977) 1440.

[6] J. R. Ellis, S. Ferrara and D. V. Nanopoulos, Phys. Lett. B 114 (1982) 231; J. Polchinski and M. B. Wise, Phys. Lett. B 125 (1983) 393; Y. Kizukuri and N. Oshimo, Phys. Rev. D 46 (1992) 3025; A. Bartl, T. Gajdosik, W. Porod, P. Stockinger and H. Stremnitzer, Phys. Rev. D 60 (1999) 073003; S. Pokorski, J. Rosiek and C. A. Savoy, Nucl. Phys. B 570 (2000) 81; T. Ibrahim and P. Nath, Phys. Rev. D 57 (1998) 478 [Erratum-ibid. D 58 (1998 ERRAT,D60,079903.1999 ERRAT,D60,119901.1999) 019901]; Phys. Rev. D 61 (2000) 093004; G. Degrassi, E. Franco, S. Marchetti and L. Silvestrini, JHEP 0511 (2005) 044.

[7] M. Endo, M. Kakizaki and M. Yamaguchi, Phys. Lett. B 583 (2004) 186.

[8] S. Dimopoulos and L. J. Hall, Phys. Lett. B 344 (1995) 185; R. Barbieri, A. Romanino and A. Strumia, Phys. Lett. B 369 (1996) 283; A. Romanino and A. Strumia, Nucl. Phys. B 490 (1997) 3; J. Hisano, M. Kakizaki, M. Nagai and Y. Shimizu, Phys. Lett. B 604 (2004) 216; J. Hisano, M. Kakizaki and M. Nagai, Phys. Lett. B 624 (2005) 239.

[9] A. Masiero, P. Paradisi and R. Petronzio, hep-ph/0511289

[10] G. Isidori and P. Paradisi, Phys. Rev. D 73 (2006) 055017.

[11] G. Isidori and A. Retico, JHEP 0111 (2001) 001; G. D'Ambrosio, G. F. Giudice, G. Isidori and A. Strumia, Nucl. Phys. B 645 (2002) 155; A. J. Buras, P. H. Chankowski, J. Rosiek and L. Slawianowska, Nucl. Phys. B 659 (2003) 3; A. Dedes and A. Pilaftsis, Phys. Rev. D 67 (2003) 015012 .

[12] G. Isidori and A. Retico, JHEP 0209 (2002) 063; J. Foster, K. Okumura and L. Roszkowski, JHEP 0508 (2005) 094.

[13] C. A. Baker et al., hep-ex/0602020

[14] M. V. Romalis, W. C. Griffith and E. N. Fortson, Phys. Rev. Lett. 86 (2001) 2505.

[15] J. Hisano and Y. Shimizu, Phys. Rev. D 70 (2004) 093001.

[16] I. B. Khriplovich and K. N. Zyablyuk, Phys. Lett. B 383 (1996) 429.

[17] P. Ciafaloni, A. Romanino and A. Strumia, Nucl. Phys. B 524 (1998) 361.

[18] G. Degrassi, P. Gambino and G. F. Giudice, JHEP 0012 (2000) 009; M. Carena, D. Garcia, U. Nierste and C. E. M. Wagner, Phys. Lett. B 499 (2001) 141.

[19] S. M. Barr, Phys. Rev. Lett. 68 (1992) 1822; O. Lebedev and M. Pospelov, Phys. Rev. Lett. 89 (2002) 101801; D. A. Demir, O. Lebedev, K. A. Olive, M. Pospelov and A. Ritz, Nucl. Phys. B 680 (2004) 339.

[20] J. Hisano and Y. Shimizu, Phys. Lett. B 581 (2004) 224. 